\title{
ORIGINAL
}

\section{LA ALIMENTACIÓN DE LOS ESCOLARES DE TRECE AÑOS DEL MUNICIPIO DE ZARAGOZA}

\author{
M. ${ }^{\mathrm{a}}$ Rosario Casado Górriz (1), Inmaculada Casado Górriz (2) y Gabriel J Díaz Grávalos (3) \\ (1) Práctica privada, Zaragoza \\ (2) Equipo Atención Primaria Allariz, Ourense. \\ (3) Centro de Salud Cea, Ourense
}

\section{RESUMEN}

Fundamento: La importancia de la dieta como causa de diversas enfermedades y la posibilidad de intervención educativa en el período escolar, hacen recomendable conocer las actitudes de los jóvenes respecto a la nutrición y sus hábitos alimentarios.

Métodos: Estudio transversal. Se realizó una encuesta autoadministrada sobre hábitos y conocimientos respecto a la nutrición, en una muestra aleatoriamente extraída de 543 escolares de $1 .^{\circ}$ de Enseñanza Secundaria Obligatoria (ESO) (13 años) de la ciudad de Zaragoza, incluyendo una valoración del consumo de alimentos mediante recuerdo de 24 horas y cuestionario de frecuencia de consumo semanal. Se realizó una prueba piloto en 50 individuos, con validación mediante entrevista personal en 15 casos.

Resultados: Se analizaron 516 cuestionarios. Un $41,5 \%$ de la muestra reconoció que la publicidad influía en su alimentación. Se encontró una mayor implicación femenina en los temas relacionados con la alimentación. La encuesta de consumo reflejaba una dieta normocalórica, muy hiperproteica y baja en carbohidratos, con exceso de lípidos en varones. Se consumía en exceso carne y derivados y dulces y golosinas, mientras se apreció un consumo bajo de cereales, pescado y patatas.

Conclusiones: Es necesario realizar actividades educativas en la edad escolar respecto a la composición de los alimentos y dieta adecuada, enseñando a mantener una actitud crítica frente a la publicidad de alimentos. Debe recomendarse una disminución de la ingesta de carnes y dulces, y un incremento del consumo de hidratos de carbono complejos y pescado.

Palabras clave: Nutrición. Adolescencia. Educación nutricional. Dieta.

\section{ABSTRACT}

\section{The Diet of Thirteen Year Old Students in the City of Saragossa}

Background: The importance of diet as a cause of different diseases and the possibility of educational involvement during the school-agc ycars leads to it being advisable to ascertain the attitudes of young people with regard to nutrition and their eating habits.

Methods: Cross-section study. A self-test survey was conducted regarding the nutrition-related habits and knowledge on a sample randomly taken from among 543 students from the $1^{\text {st }}$ level school-leaving certificate studies (age 13) in the city of Saragossa, including an assessment of the foods eaten by means of 24-hour recall and a questionnaire regarding how often different foods are eaten weekly. A pilot study was conducted on 50 individuals, with validation by means of personal interviews in 15 cases.

Results: A total of 516 questionnaires were analyzed. The subjects were found to possess an average degree of knowledge regarding nutrition-related matters. A total $41.5 \%$ of the sample acknowledged advertising having an impact on their diet. A greater diet-related impact was found to exist among the females analyzed. The eating survey revealed a diet consisting of normal calorie intakes, but excessive protein and low carbohydrate intakes, excess fat intake being noted among males. An excessive amount of Meat and meat products and Sweets and candies was noted, contrasting with the low Grain, Fish and Potato intake.

Conclusions: Educational measures must be taken among school-age children with regard to informing them concerning the composition of foods and a correct diet, teaching them to take a critical stance with regard to food advertising. They must be counseled to lessen their intakes of Meats and Sweets and to increase the amount of complex carbohydrates and Fish.

Key words: Nutrition. Adolescence. Nutrition training. Diet.
Correspondencia:

Gabriel J Díaz Grávalos

Feria, 9

32130 CEA (Ourense)

tel.: 988373461

Fax: 988282000

\section{INTRODUCCIÓN}

La dieta ha sido involucrada en los últimos años en la patogenia de diversas enfermedades, algunas de ellas causantes de gran morbilidad y mortalidad en nuestra socie$\mathrm{dad}^{1-4}$. Así, la ingesta de un número excesi- 
vo de calorías predispone a la obesidad y éste puede ser el principal factor coadyuvante de la elevación de la presión arterial $(\mathrm{PA})^{5}$, asociación que se manifiesta precozmente, pues los niños situados en los percentiles más altos de peso corporal tienen generalmente una PA más elevada ${ }^{6,7}$. El contenido calórico de la dieta y su composición influyen de manera directa sobre la tasa de lípidos circulantes ${ }^{8}$ y numcrosos estudios epidemiológicos han puesto de manifiesto que el nivel de colesterol tiene capacidad predictiva para la morbilidad y mortalidad futuras por cardiopatía isquémica, existiendo esta asociación para cualquier valor de colesterolemia ${ }^{9}$.

En los últimos años se ha detectado un incremento en los valores de lípidos en la población infantil española ${ }^{10-13}$, a la par que se han producido cambios importantes en el modelo dietético, antaño bastante favorable por un elevado consumo de cereales, arroz, pescado, aceite de oliva, legumbres y patatas $^{14,15}$, como se ha observado en diversas encuestas dietéticas llevadas a cabo en nuestro país ${ }^{16}$. A esta modificación en el patrón alimentario y su homologación con una alimentación más típica de Europa Central y Norteamérica, se le ha achacado una fuerte relación con la aparición de enfermedad vascular $^{9,17}$.

La mayor parte de los comportamientos alimentarios suelen quedar fijados en la infancia, situación que hace esta edad espccialmente adecuada para la intervención educativa en el niño y en la familia. Entre las diversas recomendaciones que el Consenso para el Control de la Colesterolemia en España realizó a comienzos de la déca$\mathrm{da}^{9}$, figura el organizar programas de educación alimentaria dirigidos a la población en general y en especial a los niños, adolescentes y jóvenes mediante la educación escolar y familiar.

Como cualquier intervención, ésta requiere un diagnóstico previo con el fin de conocer las actitudes y conocimientos de los jóvenes respecto a la nutrición, así como sus hábitos alimentarios, motivo por el que se ha realizado este estudio.

\section{MATERIAL Y MÉTODOS}

Durante el mes de mayo del año 1998, se realizó una encuesta individual sobre hábitos alimentarios y frecuencia de consumo de alimentos a una muestra de escolares de 13 años de ambos sexos que cursaban $1 .^{\circ}$ de ESO en colegios públicos y privados del municipio de Zaragoza. Los individuos se seleccionaron por medio de muestreo en conglomerados: se obtuvieron al azar 8 colegios de entre los ubicados en la ciudad, utilizándose la totalidad de la población de $1 .^{\circ}$ de ESO de cada uno de ellos (543) (tabla 1).

Tabla 1

Relación de colegios encuestados y número de alumnos aportados

\begin{tabular}{|ll|}
\hline Colegios encuestados & $N^{\circ}$ alumnos \\
\hline San Agustín & $93[18,0 \%]$ \\
Montemolin & $87[16,8 \%]$ \\
Goya & $79[15,3 \%]$ \\
San Roque & $59[11,4 \%]$ \\
Pompiliano & $58[11,2 \%]$ \\
Calasancio & $55[10,6 \%]$ \\
Montearagón & $47[9,1 \%]$ \\
Sansueña & $38[7,3 \%]$ \\
\hline
\end{tabular}

Para las variables cualitativas el tamaño muestral necesario se calculó, para una $\mathrm{p}=0,5$, un $\alpha=0,05$ y una precisión de 0,05 , en 384 individuos. Para la estimación de las variables cuantitativas se fijó una precisión de $\pm 10 \mathrm{mg}$ y un $\alpha=0,05$. De estudios previos $^{18}$, se obtuvo el valor de la desviación estándar de la ingesta de kilocalorias totales, $\mathrm{s}=630 \mathrm{mg}$, y con esos datos se calculó un tamaño de muestra de 153 individuos.

La encuesta fue administrada con carácter anónimo y voluntario, a lo largo del mes de mayo de 1998, a través del tutor de cada cla- 
se, dentro del horario lectivo y a los alumnos presentes en ese momento. Previamente a su realización se instruyó a los alumnos sobre la finalidad de la misma, su condición de anónima y la forma de cumplimentarla. No hubo negativas a participar, siendo el porcentaje de ausencias del 2,7\% (15), debidas a no asistencia a clase ese día, fundamentalmente por enfermedad. En total fueron obtenidos 516 cuestionarios válidos, 276 varones y $240 \mathrm{mu}-$ jeres. Doce $(2,3 \%)$ fueron descartados por mala cumplimentación.

El cuestionario inicial había sido probado con 50 alumnos de otro centro no seleccionado para este estudio. La parte que exploraba las actitudes y conocimientos de los escolares fue validada mediante entrevista personal con 15 de los encuestados y posterior comparación entre ambos métodos, quedando constituida definitivamente por 25 ítems que indagaban, entre otros, hábitos y horarios de comida (2), compra y elaboración de alimentos (2), conocimientos generales sobre alimentación (7), influencia de la publicidad (2) y presencia de hábitos tóxicos (2), así como edad y sexo (Anexo 1). Adicionalmente se realizó una encuesta de consumo de alimentos dividida en dos partes: encuesta de frecuencia de consumo semanal y recuerdo de 24 horas. A partir de la encuesta de frecuencia de consumo semanal se obtuvieron las cantidades de alimentos ingeridas, considerando las raciones de acuerdo con los datos obtenidos en el estudio CAENPE de la Comunidad Autónoma de Madrid ${ }^{18}$ y los resultados del recuerdo de 24 horas incluido en el cuestionario. La determinación de las ingestas de energía, principios inmediatos, fibra, minerales y vitaminas, así como de colesterol total y ácidos grasos se realizó a partir de las Tablas de Composición de Alimentos modificadas del $\mathrm{CSIC}^{19}$. El alcohol no fue incluido en el cálculo energético. Las ingestas diarias recomendadas usadas como comparación fueron las calculadas por el Instituto de $\mathrm{Nu}$ trición del CSIC en $1994^{20}$.

Los datos se analizaron con ayuda de los programas estadísticos SPSS/PC+ y
EPIDAT, utilizándose estimación de porcentajes para la descripción de las variables cualitativas (conocimientos y hábitos sobre nutrición) y estimación de medias para los datos cuantitativos referidos a ingestas $y$ consumos, con sus correspondientes intervalos de confianza del $95 \%\left(\mathrm{IC}_{95 \%}\right)$. La diferencia entre grupos se cuantificó mediante el cálculo de la razón de proporciones.

\section{RESULTADOS}

Se tabuló un total de 516 cuestionarios, 276 varones y 240 mujeres. La media de edad de los sujetos estudiados estaba en 12,5 años (Desviación Estándar [DE] 0,5), con un valor mínimo de 11 años y un máximo de 14.

El $89 \%\left(\mathrm{IC}_{95 \%}[85,8-91,5]\right)$ de los encuestados refería realizar al menos una comida diaria en familia, realizandolas a horas fijas el $83 \%\left(\operatorname{IC}_{95 \%}[79,4-86,0]\right)$.

La colaboración en la compra de alimentos se daba en un $84 \%$ de los indivi duos $\left(\mathrm{IC}_{95 \%}[80,4-86,9]\right)$, con una diferencia de 10 puntos a favor del sexo femenino (Razón de Proporciones: 2,13: $\mathrm{IC}_{95 \%}[1,25-$ $3,63]$. El 62,5\% de los encuestados ( IC $_{95 \%}$ $[58,1-66,6])$ participaba de forma regular en la elaboración de alimentos en el domicilio, apreciándose una diferencia de 22 puntos entre mujeres $(74 \%)$ y varones $(52 \%)$ ( Razón de Proporciones 2,65: $\mathrm{IC}_{95 \%} \quad[1,79-$ 3,92]).

Un $41,5 \%\left(\mathrm{IC}_{95 \%}[37,2-45,8]\right)$ de la muestra reconocía que la publicidad de alimentos o bebidas en la televisión influye en su consumo; mientras, el $65 \%\left(\operatorname{IC}_{95 \%}[60,6-69,0]\right)$ consideraba que la comida de casa es más sana que la que se consume en establecimientos de comida rápida (hamburgueserías, pizzerías, etc.).

El $87 \%\left(\operatorname{IC}_{95 \%}[83,7-89,7]\right)$ de la muestra afirmaba preocuparse por llevar una dieta adecuada y un $96 \%\left(\mathrm{IC}_{95 \%}[93,8-97,4]\right)$ creía entender lo que es una alimentación equili- 
brada; el 61,5\% (IC $\left.\mathrm{IS}_{95 \%}[57,1-65,6]\right)$ afirmaba preocuparse de obtener información acerca de los alimentos más convenientes, conociendo la rueda de alimentos un $62,5 \%$ $\left(\mathrm{IC}_{95 \%}[58,1-66,6]\right)$ y las tablas de composición de alimentos el 38,5\% ( $\mathrm{IC}_{95 \%}$ [34,342,8]) (tabla 2).
La tabla 3 muestra los consumos referidos en los diferentes grupos de alimentos junto a la Raciones Diarias Recomendadas (RDR).

En la tabla 4 quedan reflejadas las ingestas diarias medias de calorías, principios inmediatos, fibra, calcio, hierro y diversas vi-

Tabla 2

Principales resultados del cuestionario sobre hábitos y conocimientos en nutrición (Expresados en porcentaje de respuesta afirmativa junto al $\mathrm{IC}_{\mathbf{9 5} \%}$ )

\begin{tabular}{|lrrr|}
\hline Hábitos y conocimientos & \multicolumn{1}{c}{ Varones } & \multicolumn{1}{c|}{ Mujeres } & Total \\
\hline Comen habitualmente en familia & $86,5[81,7-90,1]$ & $92[87,6-94,9]$ & $89[85,8-91,5]$ \\
Comen a horas fijas & $85,5[80,6-89,3]$ & $81[75,3-85,6]$ & $83,5[79,9-86,5]$ \\
Colaboran en la compra de alimentos & $79,5[74,1-84,0]$ & $89[84,1-92,5]$ & $84[80,4-86,9]$ \\
Colaboran en la elaboración de comidas & $52[45,9-58,0]$ & $74[67,8-79,3]$ & $62,5[58,1-66,6]$ \\
Influye la publicidad en los alimentos que consumen & $40,5[34,7-46,5]$ & $43,5[37,1-50,0]$ & $41,5[37,2-45,8]$ \\
Afirman comprender el significado de una alimenta- & & & \\
$\quad$ ción equilibrada & $94,5[90,9-96,7]$ & $97,5[94,3-98,9]$ & $96[93,8-97,4]$ \\
Se preocupan por lo que comen & $85[80,1-88,8]$ & $89,5[84,7-92,9]$ & $87[83,7-89,7]$ \\
Conocen la rueda de los alimentos & $61,5[55,4-67,2]$ & $63,5[57,0-69,5]$ & $62,5[58,1-66,6]$ \\
Conocen las tablas de composición de alimentos & $41[35,1-47,0]$ & $36[29,9-42,4]$ & $38,5[34,3-42,8]$ \\
Consideran la «comida rápida» respecto a la de casa & & & $35[30,9-39,3]$ \\
$\quad$ igual o mejor & $36[30,3-42,0]$ & $34[28,1-40,4]$ & 35 \\
\hline
\end{tabular}

Tabla 3

Consumos referidos de los principales grupos de alimentos (Datos obtenidos a partir de la encuesta de frecuencia de consumo semanal)

\begin{tabular}{|c|c|c|c|c|}
\hline \multirow{2}{*}{ Grupos de alimentos } & \multicolumn{2}{|c|}{ Total } & \multirow{2}{*}{$\begin{array}{c}\text { Varones } \\
{[g / p / d \pm D E]}\end{array}$} & \multirow{2}{*}{$\begin{array}{c}\text { Mujeres } \\
{[g / p / d \pm D E]}\end{array}$} \\
\hline & {$[g / p / d \pm D E]$} & [R.D.R.] & & \\
\hline Carne y derivados & $199 \pm 57$ & [85] & $205 \pm 61$ & $189 \pm 55$ \\
\hline Pescados & $76 \pm 46$ & [115] & $71 \pm 45$ & $82 \pm 46$ \\
\hline Verduras & $87 \pm 50$ & {$[110]$} & $85 \pm 50$ & $88 \pm 49$ \\
\hline Hortalizas & $34 \pm 7^{\#}$ & & $31 \pm 9$ & $38 \pm 9$ \\
\hline Patatas & $48 \pm 30$ & {$[120]$} & $49 \pm 31$ & $46 \pm 29$ \\
\hline Legumbres & $29 \pm 15$ & {$[35]$} & $31 \pm 15$ & $27 \pm 16$ \\
\hline Cereales & $188 \pm 48$ & {$[310]$} & $203 \pm 49$ & $174 \pm 48$ \\
\hline Huevos & $27 \pm 11$ & {$[30]$} & $27 \pm 12$ & $27 \pm 11$ \\
\hline Leche y derivados & $545 \pm 87$ & {$[600]$} & $561 \pm 88$ & $539 \pm 84$ \\
\hline Frutas & $332 \pm 89$ & {$[275]$} & $341 \pm 95$ & $319 \pm 79$ \\
\hline Bebidas & $79 \pm 64$ & & $81 \pm 64$ & $77 \pm 63$ \\
\hline Dulces y golosinas * & $96 \pm 35$ & {$[60]$} & $100 \pm 35$ & $90 \pm 36$ \\
\hline Grasas $^{\dagger}$ & $27 \pm 11$ & {$[50]$} & $28 \pm 12$ & $25 \pm 11$ \\
\hline Alimentos procesados ${ }^{\circ}$ & $53 \pm 17$ & & $59 \pm 19$ & $48 \pm 21$ \\
\hline
\end{tabular}

g/p/d: gramos por persona y día.

R.D.R. = ración diaria recomendada.

*: Azúcar, bollería, pastelería caramelos, chocolate, galletas, golosinas, mermeladas, ganchitos y similares.

$\dagger$ : Aceites, mantequilla, margarina, mayonesa, nata.

: Precocinados como croquetas, empanadillas, varitas, etc.

": R. D. R. incluida en Verduras. 
Tabla 4

Ingesta referida de calorías, principios inmediatos, fibra, calcio, hierro y diversas vitaminas, frente a ingestas recomendadas (Datos obtenidos a partir de la encuesta de frecuencia de consumo semanal)

\begin{tabular}{|c|c|c|c|c|}
\hline & Varones & $\begin{array}{l}\text { Recomendad } \\
\text { en varones }\end{array}$ & Mujeres & $\begin{array}{c}\text { Recomendado } \\
\text { en mujeres }\end{array}$ \\
\hline Energía $(\mathrm{Kcal} \pm \mathrm{DE})$ & $2.677 \pm 635$ & 2.600 & $2.490 \pm 487$ & 2.400 \\
\hline Proteínas $(\mathrm{g} \pm \mathrm{DE})$ & $123 \pm 31$ & 45 & $117 \pm 32$ & 43 \\
\hline$\%$ E. A. ${ }^{*}$ & 18,4 & 6,9 & 18,8 & 7,1 \\
\hline Lípidos $(\mathrm{g} \pm \mathrm{DE})$ & $105 \pm 17$ & $87^{\ddagger}$ & $86 \pm 13$ & $80^{\ddagger}$ \\
\hline$\%$ E. A. ${ }^{*}$ & 35,3 & 30,1 & 31,1 & 30,0 \\
\hline $\mathrm{H}$ de $C^{\dagger}(\mathrm{g}+\mathrm{DE})$ & $310 \pm 66$ & 409 & $312 \pm 57$ & 377 \\
\hline$\%$ E. A. ${ }^{*}$ & 46,3 & 62,9 & 50,1 & 62,8 \\
\hline Calcio (mg) & 1.450 & 1.000 & 1.350 & 1.000 \\
\hline Hierro (mg) & 16,0 & 13,5 & 15,0 & 18,0 \\
\hline Fibra (mg) & 23 & 15 & 20 & 15 \\
\hline Vitamina $\mathrm{B}_{2}(\mathrm{mg})$ & 2,4 & 1,6 & 2,4 & 1,5 \\
\hline Niacina (mg) & 51 & 17 & 46 & 16 \\
\hline Vitamina $\mathrm{C}(\mathrm{mg})$ & 92 & 60 & 89 & 60 \\
\hline Retinol $(\mu \mathrm{g})$ & 972 & 1.000 & 950 & 800 \\
\hline Vitamina $\mathrm{D}(\mu \mathrm{g})$ & 13,0 & 5,0 & 12,2 & 5,0 \\
\hline
\end{tabular}

*: Porcentaje de energía aportado.

$\dagger$ : Hidratos de Carbono.

†: Máximo recomendado (30\% ingesta calórica)

taminas, así como las ingestas diarias recomendadas en función del sexo.

Cabe destacar que el $27 \%$ de la energía consumida procede de los cereales, el $20 \%$ de la leche y derivados y un $12 \%$ de la carne. El $29 \%$ de las proteínas proceden de la carne, un $25 \%$ de la leche, el $15 \%$ de los cereales y un $11 \%$ del pescado.

El mayor aporte de lípidos viene de la leche y derivados $(30 \%)$, un $21 \%$ de las grasas y un $17 \%$ de la carne. El aporte de co- lesterol total supone $288 \mathrm{mg}$ por día de promedio. La tabla 5 muestra los aportes de los diferentes tipos de ácidos grasos de la dieta frente a las ingestas recomendadas.

Los cereales suponen el 49\% de los hidratos de carbono y el $28 \%$ de la fibra.

\section{DISCUSIÓN}

Se eligió como método de encuesta para el cálculo de las ingestas la combinación de un

Tabla 5

Ingesta obtenida frente a la recomendada de los diferentes tipos de ácidos grasos (Datos obtenidos a partir de la encuesta de frecuencia de consumo semanal)

\begin{tabular}{|lccc|}
\hline & \multicolumn{3}{c|}{ Ingesta de ácidos grasos } \\
\cline { 2 - 4 } & Total & $\%$ cal ${ }^{*}$ & $\%_{\text {cal rec }}{ }^{+}$ \\
\hline Acidos grasos poliinsaturados & $18,4 \mathrm{~g}$ & $6,6 \%$ & máximo $10 \%$ \\
Acidos grasos monoinsaturados & $40,5 \mathrm{~g}$ & $14,6 \%$ & 10 a $15 \%$ \\
Acidos grasos saturados & $33,1 \mathrm{~g}$ & $11,9 \%$ & máximo $10 \%$ \\
\hline
\end{tabular}

*: \% sobre calorías totales de la dieta.

':\% recomendado sobre calorias totales. 
recuerdo de 24 horas con un cuestionario de frecuencia de consumo semanal, de acuerdo con la recomendación de que una combinación de ambos puede ofrecer un panorama bastante preciso del consumo poblacional $^{21,22}$. Igualmente, se consigue así incluir un día festivo, ya que existen diferencias en la ingesta según el día de la semana ${ }^{21,23}$.

Se decidió restringir el estudio al grupo de edad correspondiente a 13 años, ya que creemos que a esta edad el niño es capaz de opinar sobre sus hábitos alimentarios y que niños más pequeños necesitan la colaboración de un adulto para la cumplimentación del cuestionario ${ }^{21}$.

Aunque la muestra se obtuvo mediante un proceso aleatorio, no se ponderó respecto a la población de los diferentes barrios o distritos del término municipal, hecho a tener en cuenta al valorar su representatividad, puesto que, como en la mayor parte de las ciudades, la población infantil se encuentra desigualmente repartida entre los diferentes distritos.

La mayoría de los encuestados realizaba alguna de las comidas principales en el ámbito familiar y con regularidad horaria. De todos modos, hasta un $11 \%$ comía habitualmente fuera del núcleo familiar, situación importante en tanto que favorece la aparición de hábitos alimentarios negativos, e incluso puede perjudicar de forma global la salud del adolescente ${ }^{24}$.

Existe una mayor implicación del sexo femenino en la compra y preparación de alimentos, quizá respondiendo al papel tradicional de la mujer en este ámbito, aunque otros estudios han mostrado mayor preocupación femenina por la alimentación ${ }^{25}$.

La publicidad llega a influir en más del $40 \%$ de la población en la elección de los alimentos. Esta cifra es realmente importante considerando que, con frecuencia, los productos alimenticios publicitados se corresponden con un patrón poco recomendable, con abundantes calorías y azúcares, es- casa fibra y presencia de grasas satura das $^{25,26}$. Por motivo similar, resulta igualmente preocupante el hecho de que un $35 \%$ de la población crea que la comida de hamburgueserías y similares es, al menos, tan sana como la comida casera.

Existe un aceptable nivel de interés respecto a la alimentación, aunque el conocimiento de la clasificación y composición de los alimentos, así como las posibilidades de sustitución entre los mismos son más reducidos y claramente mejorables, como se aprecia en estudios similares en otras zonas del país ${ }^{25,27}$.

Las ingestas referidas deben valorarse considerando el efecto estacional sobre el consumo de alimentos. En este caso se corresponde con la dieta consumida en la fecha de realización del estudio, finales de primavera. Es probable que en otros momentos del año sea sustancialmente diferente, principalmente en determinados grupos de alimentos tales como frutas ${ }^{28}$.

El análisis de los consumos muestra una dieta normocalórica, muy hiperproteica y baja en carbohidratos, con exceso de ingesta de lípidos entre los varones. La comparación con otras encuestas ${ }^{18,29-34}$ muestra diferencias importantes, sobre todo en calorías totales. Así, en la Comunidad de Madrid ${ }^{18}$ se obtuvieron $2800 \mathrm{Kcal} /$ día frente a las 2.200 de Reus $^{34}$ y las 2.500 obtenidas en la presente. La comparación con otros estudios es más compleja por presentar los datos agrupados o no incluir este grupo de edad. Respecto a la ingesta de principios inmediatos, la tabla 6 muestra las diferencias entre estos tres estudios. Aunque fue realizado en adultos, debido a la influencia de los hábitos familiares en la alimentación infantil ${ }^{35}$, creemos que merece la pena destacar la coincidencia cualitativa con un reciente estudio de Ariño et al, realizado en Aragón $^{36}$, en el que se aprecia un aporte elevado de proteínas y grasas y bajo de carbohidratos. 
Tabla 6

\begin{tabular}{|c|c|c|c|}
\hline \multicolumn{4}{|c|}{$\begin{array}{l}\text { Comparación de las ingestas de principios inmediatos en } \\
\text { niños de } 13 \text { años obtenidas en diferentes estudios } \\
\text { nutricionales }\end{array}$} \\
\hline & $\begin{array}{l}\text { C.A. Ma- } \\
\text { drid (91) }\end{array}$ & $\begin{array}{c}\text { Reus } \\
\text { (92/93) }\end{array}$ & $\begin{array}{c}\text { Zaragoza } \\
\text { (98) }\end{array}$ \\
\hline Proteínas & $115 \mathrm{~g}$ & $82 \mathrm{~g}$ & $120 \mathrm{~g}$ \\
\hline Grasas & $135 \mathrm{~g}$ & $100 \mathrm{~g}$ & $95 \mathrm{~g}$ \\
\hline Hidratos de carbono & $282 \mathrm{~g}$ & $220 \mathrm{~g}$ & $310 \mathrm{~g}$ \\
\hline
\end{tabular}

La ingesta de colesterol total se encuentra por debajo del recomendado $(300 \mathrm{mg})^{8}$, con valores muy inferiores a los $390 \mathrm{mg}$ encontrados en Reus $^{34}$ o los $500 \mathrm{mg}$ del estudio CAENPE $^{18}$. Sin embargo, el análisis de la distribución de los ácidos grasos muestra exceso de grasas saturadas que se correlacionan epidemiológicamente con una alta incidencia de cardiopatía isquémica ${ }^{17,37}, y$ que se han encontrado igualmente en otros estudios $^{15,18,30,34}$. Existen en este caso escasas diferencias cuantitativas con los datos de las encuestas de Madrid y Reus, observándose en ambas un exceso de grasas saturadas que es máximo en la Comunidad de Madrid.

En alguno de estos parámetros, parte de las diferencias probablemente puedan explicarse por variaciones metodológicas, ya se ha apuntado en algún análisis reciente $^{16,38}$.

Por grupos de alimentos, se observó un elevado consumo de carne y derivados, que supone más del doble de la ración recomendada, así como del grupo correspondiente a dulces y golosinas. Había un bajo consumo de cereales, pescado y patatas. En concreto, éstas se sitúan muy por debajo de lo recomendado, aunque con valores superiores a los de otros estudios ${ }^{18,34,39-41}$. El alto consumo de fruta podría corresponder, como ya se ha indicado, a un efecto estacional. Destaca el consumo de leche y derivados, cercano a la RDR, y claramente superior al de los estudios similares citados.
Ante estos resultados creemos que es necesario incrementar los esfuerzos educativos en nutrición en este grupo etario, reforzando los conocimientos sobre composición de los alimentos, posibilidades de sustitución entre ellos e importancia de una dieta adecuada en el mantenimiento de la salud. Es necesario favorecer una actitud crítica hacia la publicidad, enseñando a valorar la composición de los alimentos y a rechazar aquéllos que no respondan a criterios de adecuación ${ }^{9}$.

Específicamente, sería necesario recomendar una disminución de la ingestión de carne y derivados y dulces y golosinas, y un incremento del consumo de cereales, pescado y patatas.

\section{BIBLIOGRAFÍA}

1. Banegas JR, Rodríguez F, Martín J, del Rey J. Comer menos grasa para prevenir la Cardiopatía Isquémica. Impacto potencial de las estrategias poblacional e individual de control de la colesterolemia en España. Med Clin (Barc) 1993; 101: 81-86.

2. Cox Ch, Mann J, Sutherland W, Ball M. Individual variation in plasma cholesterol response to dietary saturated fat. BMJ 1995; 311: 1260-1264.

3. Mata P, de Oya M, Pérez-Jiménez F, Ros E. Dieta y enfermedades cardiovasculares. Recomendaciones de la Sociedad Española de Arteriosclerosis. Clin Invest Arteriosel 1994; 6: 43-61.

4. Serra L1, Ribas L, Castell C, Arija V, Lloveras G, Salleras L. Consejo nutricional y alimentario. Me:d Clin (Barc) 1994; 102(Supl 1):93-99.

5. Stevens VJ. Weight loss intervention in phase 1 of the Trials of Hypertension Prevention. Arch Intern Med 1993; 153: 849-858.

6. National Research Council. Diet and Hcalth. Implications for reducing chronic disease risk. Washington: National Acadcmy Press;1989.

7. Elcarte R, Villa I, Sada J, Gasco M, Oyarzábal M, Sola A et al. Estudio de Navarra (PECNA). Correlación de presión arterial, en una población infanto-juvenil, con parámetros antropométricos y bioquímicos. An Esp Pediatr 1993; 39: 5-9.

8. Grundy SM, Denke MA. Dietary influences on serum lipids. J Lipid Res 1990;31: 1149-1172. 
9. Ministerio de Sanidad y Consumo. Consenso para el Control de la Colesterolemia en España. 2. ${ }^{a}$ ed. Madrid: Ministerio de Sanidad y Consumo; 1991 .

10. Elcarte R, Villa I, Sada J, Gasco M, Oyarzábal M, Solá A et al. Estudio de Navarra (PECNA). Prevalencia de hipertensión arterial, hiperlipidemia y obesidad en población infantil de Navarra. Asociación de factores de riesgo. An Esp Pediatr 1993; 38: 428-436.

11. Elcarte R, Villa I, Sada J, Gasco M, Oyarzábal M, Solá A et al. Estudio de Navarra (PECNA). Hiperlipidemia IV. Prevalencia de hiperlipidemia en la población infantil y juvenil de Navarra. Variaciones según edad, sexo y accesibilidad a la atención sanitaria. An Esp Pediatr 1993; 38: 205-212.

12. Sánchez M, González A, García B, Santos M, Serna C, Arias M et al. Patrón lipídico de niños y adolescentes de Madrid. An Esp Pediatr 1992; 37: $205-210$.

13. Prieto L, Mateos C, Galán A, Arroyo J, Vadillo JM. Perfil lipídico en niños y adolescentes de la provincia de Cáceres. Aten Primaria 1999; 23: 404-410.

14. Varela G, Moreiras O, Carbajal A, Campo M. Estudio nacional de nutrición y alimentación 1990/91 (ENNA-3). Madrid: Instituto Nacional de Estadística; 1995.

15. Medrano MJ, Almazán J, Sierra MJ, Olalla MT. Situación epidemiológica de las enfermedades cardiovasculares arterioscleróticas (II): prevalencia de factores de riesgo. Bol Epidemiol Semanal 1998; 6: 157-164.

16. Gorgojo L, Guallar E, Martín-Moreno JM, Iópez-Nomdedeu C, Vázquez C, Martí-Henneberg $\mathrm{C}$, Serrano-Ríos M. Encuestas alimentarias en los niños españoles de edad escolar: análisis del período 1984-1994. Med Clin (Barc)1999; 112: 368-374.

17. Posner B, Cobb JL, Belanger A, Cupples LA, D'Agostino RB, Stokes J. Dietary lipid predictors of coronary heart disease in men. Arch Intern Med 1991; 151: 1181-1187.

18. Subdirección General de Higiene de los Alimentos (estudio CAENPE). Consumo de alimentos y estado nutricional de la población escolar de la Comunidad Autónoma de Madrid (estudio CAENPE). Madrid: Ministerio de Sanidad y Consumo; 1994.

19. García Diz L. Nutrición y dietética [programa informático]. Versión MS-DOS. Madrid: Universidad Complutense de Madrid; 1994.
20. Instituto de Nutrición (CSIC). Recomendaciones de Energía y Nutrientes para la población española. Madrid: Instituto de Nutrición (CSIC); 1994.

21. Banegas JR, Villar F, Gil E, Carretero ML, Arranz I, Aranceta J et al. Directrices para la elaboración de estudios poblacionales de alimentación y nutrición. Rev San Hig Púb 1994; 68: 247-260.

22. Barrio J, Román E, Cilleruelo ML. Revisión de métodos para análisis de ingesta alimentaria individual: La cncuesta alimentaria. Pediátrika 1999; 19: $79-87$.

23. Tood KS, Hudes M, Caloway DH. Food intake measurement: problems and approaches. Am J Clin Nutr 1983; 37: 139-146.

24. Compañ E, Moreno J, Pascual E, Ruiz MT, Belda P. Estilo de vida familiar y salud de los adolescentes. Aten Primaria 1998;22(Supl 1): 324.

25. Goñi C, Vilches C, Ancizu E, Arillo A, Lorenzo V, Ancizu P et al. Factores relacionados con los comportamientos alimentarios en una población juvenil urbana. Aten Primaria 1999; 23: 32-37.

26. Ortega RM, Andrés P, Jiménez LM, González-Gross M, Ortega A. Mensajes publicitarios de contenido nutricional dirigidos a la población infantil por televisión. Nutr Clin Diet Hosp 1993; 13: $25-34$

27. Martínez JM, Rodríguez A, Ruiz MA, Díaz MC, Rendón MA, Cano C et al. Nivel de conocimientos sobre alimentación/nutrición en adolescentes escolarizados de Cádiz. Aten Primaria 1998; 22: 33-38.

28. Salas J, Font I, Canals J, Guinovart L, Sospedra $\mathrm{C}$, Martí $\mathrm{C}$. Consumo, hábitos alimentarios y estado nutricional de la población de Reus:(I) Consumo global por grupos de alimentos y su relación con el nivel socioeconómico y de instrucción. Med Clín (Barc) 1985; 84: 339-343.

29. Jiménez CP, Rodríguez M, López JJ, Ibáñez A, Gámez E, Odriozola G. Estudio nutricional completo en escolares, opiniones y actitudes. Aten Primaria 1998; 22 (Supl 1): 314.

30. Failde I, Zafra JA, Ruiz E, Novalbos JP. Valoración de la alimentación de los escolares de una población de la Sierra de Cádiz (Ubrique). Med Clin (Barc) 1997; 108: 254-258.

31. Pena G, Fcrnández-Crehuet MN, Villanueva E, Ruiz JV, Vázquez MA. Hábitos alimentarios entre la población escolar del medio rural. Aten Primaria 1996; 18: 452-456. 
32. Chaves M, Santodomingo F, Muradas M, Fornos JA. Estudio de la ingesta alimentaria de un colectivo homogéneo de niños de ambos sexos y de edades comprendidas entre seis y quince años. Nutr Clín 1990; 10: 49-51.

33. Mur de Frenne L, Fleta J, Moreno L. Ingesta de alimentos a lo largo del día en niños zaragozanos. Nutr Clin 1994; 14: 57-68.

34. Arija V, Salas J, Fernández-Ballart J, Cucó G, Martí-Henneberg C. Consumo, hábitos alimentarios y estado nutricional de la población de Reus (VIII). Evolución de la ingestión de energia y nutrientes entre 1983 y 1993. Med Clin (Barc) 1996; 106: $45-50$.

35. Rodríguez L, Martinez E, Machín M, Sánchez M. Influencia de los aspectos higiénico-culturales del entorno familiar en los patrones dietéticos del niño escolar. Med Clin (Barc) 1994;102:1-4.

36. Ariño A, Pérez C, Lázaro R, Herrera A. Estudio preliminar de los hábitos alimentarios y el estado nutricional en una muestra de la población aragonesa. Alim Nutri Salud 1998; 5: 33-40.

37. Carmena R, Ordovás JM. Hiperlipemias. Clínica y tratamiento. Barcelona: Ediciones Doyma; 1999.
38. Rodríguez-Artalejo F, Graciani MA, Banegas JR, Martín-Moreno JM, Sabaté J, Rey-Calero J. El consumo de alimentos y nutrientes en España en el período 1940-1988. Un estudio comparativo de las principales fuentes de información sobre consumo alimentario. En: de Oya M, Garcés C, editores. Metabolismo lipídico. Madrid: IDEPSA; 1997: $245-258$.

39. Vázquez C, de Cos AI, Martinez P, Jaunsolo MA, Roman E, Gómez C et al. Consumo de alimentos y estado nutricional de los escolares de la Comunidad de Madrid (CAENPE): metodologia general y consumo global de alimentos. Nutr Hosp 1995; 10: 40-48.

40. Martínez P, Jaunsolo MA, Fernández C, Roman $\mathrm{E}$, López C, Vázquez C. Ingesta de alimentos de procedencia animal distintos de la leche: carnes, pescados y huevos, en la población escolar de la Comunidad de Madrid. Grupo CAENPE. An Esp Pediatr 1996; 44: 209-213.

41. Fernández C, López T, Martínez P, Jaunsolo MA, De Cos AI, Cilleruelo ML et al. Consumo de lácteos y su contribución al aporte de nutrientes en la dieta de los escolares de la Comunidad de Madrid. Grupo CAENPE. An Esp Pediatr 1996; 44: 214-218. 


\section{Anexo 1}

\section{Cuestionario referido a hábitos y conocimientos sobre nutrición}

1. Habitualmente, ¿realizas alguna de las comidas del día con tu familia?

Sí No Ns/nc

2. ¿Comes o cenas habitualmente a la misma hora?
Si
No
$\mathrm{Ns} / \mathrm{nc}$

3. ¿Compras o ayudas a comprar algún alimento para tu familia?

\begin{tabular}{lll}
$\mathrm{Si}$ & $\mathrm{No}$ & $\mathrm{Ns} / \mathrm{nc}$ \\
\hline
\end{tabular}

4. ¿Participas alguna vez en la elaboración de comidas en tu casa?
Sí
No
$\mathrm{Ns} / \mathrm{nc}$

5. ¿Comes o bebes los alimentos que ves en los anuncios?
Sí
No
$\mathrm{Ns} / \mathrm{nc}$

6. ¿Los comerías si no se anunciaran?
$\mathrm{Si}$
No
$\mathrm{Ns} / \mathrm{nc}$

7. ¿Sabes lo que significa alimentación equilibrada?
Sí
No
$\mathrm{Ns} / \mathrm{nc}$

8. ¿Te has informado alguna vez de qué alimentos son los más convenientes?
Sí
No
$\mathrm{Ns} / \mathrm{nc}$

9. ¿Te preocupa cuidar bien tu alimentación?
Sí
$\mathrm{No}$
$\mathrm{Ns} / \mathrm{nc}$

10. ¿Sabes lo que es la rueda de los alimentos?
Sí
No
$\mathrm{Ns} / \mathrm{nc}$

11. ¿Sabes lo que son las tablas de composición de los alimentos?
Sí
No
$\mathrm{Ns} / \mathrm{nc}$

12. ¿Cómo consideras los alimentos de las hamburgueserías, pizzerías y similares respecto a la comida de casa? Igual mejor peor

13. ¿Te gustaría elaborar un plan nutricional para mejorar tu alimentación?
Sí
No
$\mathrm{Ns} / \mathrm{nc}$ 